\title{
FORMULATION DEVELOPMENT OF IMMEDIATE RELEASE PELLETS OF TADALAFIL: SOLIDIFICATION APPROACH FOR NANOSUSPENSION
}

\author{
SHOBHA UBGADE*, VAISHALI KILOR, VIDYA BAHEKAR, ABHAY ITTADWAR
}

Gurunanak College of Pharmacy, Dixit Nagar, Nagpur, India

Email: shobha_yadav1402@yahoo.co.in

Received: 01 Feb 2019, Revised and Accepted: 26 Apr 2019

\begin{abstract}
Objective: Nanosuspension is known to enhance the saturation solubility and dissolution velocity of poorly soluble drugs owing to the increased surface area of nanosized particles. Stability of these solubility enhancing systems can be improved by converting them into solidified forms. To simultaneously achieve enhanced dissolution and improved stability, an attempt has been made to increase the dissolution rate of poorly soluble drug tadalafil by formulating immediate release pellets of its nanosuspension.

Methods: Tadalafil nanosuspensions were prepared using high shear homogenization technique and hydroxypropyl methylcellulose (HPMC) E 15, sodium dodecyl sulphate (SDS) as stabilizers. Prepared nanosuspensions were subjected to the characterization of particle size distribution, zeta potential, drug loading and saturation solubility. Optimized nanosuspension was solidified by preparing immediate release pellets: for improved stability, where tadalafil nanosuspension was used as a binder. Pellets were prepared by extrusion-spheronization technique using $\kappa$-carrageenan as a pelletizing aid.
\end{abstract}

Results: Prepared immediate release pellets disintegrated within $03 \mathrm{~min}$. In vitro dissolution studies showed $85 \%$ drug release within 45 min in pH 1.2 buffer from immediate release pellets containing tadalafil nanosuspension.

Conclusion: It can be concluded that formulation of nanosuspension of poorly soluble drug and its use as a binder for the preparation of immediate release pellets markedly improved the dissolution rate.

Keywords: Tadalafil, Nanosuspension, Immediate release, Dissolution enhancement, Solidification

(C) 2019 The Authors. Published by Innovare Academic Sciences Pvt Ltd. This is an open-access article under the CC BY license (http://creativecommons.org/licenses/by/4.0/) DOI: http://dx.doi.org/10.22159/ijap.2019v11i4.32331

\section{INTRODUCTION}

One of the major challenges of the pharmaceutical researchers is to develop an enabling formulation which can make poorly water-soluble drugs highly soluble to overcome the low bioavailability problem of the drugs. Many strategies are used to improve the aqueous solubility of the poorly soluble drugs such as complexation [1], micronisation [2], lipid based formulations, use of cosolvents, solid dispersions [3] etc. However, these techniques often lead to poor solubility, toxicity [4], high production costs [5] and therefore fails at commercial level.

Nanotechnology-based formulations have been valuable in improving the solubility of poorly soluble drugs, as nanosuspension formulations have shown good results in many cases in improving saturation solubility due to the increased surface area available for dissolution [6,7]. Literature reports use of high shear homogenization for the preparation of oral nanosuspension formulations of some poorly water-soluble drugs. Since nanosuspensions suffer from stability problems like aggregation, sedimentation, crystalline transformation, nanosuspension can be solidified by using spray drying method [8], fluid bed drying [9] or by converting into oral thin film [10] formulation. However, these methods may lead to the increased cost of the product. Thus, an approach is required that is not only providing solubility improvement but also imparting stability to the formulation during shelf life and also which involves simple techniques that can be easily employed for commercial production.

Pelletization is an agglomeration process that converts fine powders or granules of bulk drugs and excipients into small, free-flowing, spherical or semi-spherical units, referred to as pellets. Pellets range in size; typically, between $0.5 \mathrm{~mm}$ and $1.5 \mathrm{~mm}$. Pellets being smaller in size offer a larger surface area for dissolution than any other solid dosage form [11]. The surface area can further be increased by making these pellets disintegrate immediately. Decreasing the particle size of poorly soluble drugs is an established approach for improving solubility, thus improving bioavailability [12].

In the present research work, tadalafil was used as a model drug and its nanosuspensions were prepared using high shear homogenization method for the dissolution improvement. Optimized nanosuspension was used as a binder for the preparation of immediate release (IR) pellets, where, $\mathrm{K}$-carrageenan was used as a pelletizing agent [13].

The objective of the study was to investigate the feasibility of using high shear homogenizer for the preparation of nanosuspension and use of drug-loaded nanosuspension as a binder for the preparation of immediate release pellets in order to improve the dissolution rate of model drug tadalafil. Tadalafil is a BCS Class II drug and possess poor solubility. It is primarily used to treat erectile dysfunction, benign prostate hyperplasia and primary pulmonary hypertension [14]. High selectivity and better efficacy, makes it a drug of choice for the treatment of erectile dysfunction.

Physicochemical properties of nanosuspension including particle size, zeta potential, and saturation solubility were evaluated. IR pellets were prepared using $\mathrm{k}$-carrageenan as a pelletizing agent. Since, the disintegration of pellets will lead to increase in surface area of the drug which is already in nanosize, therefore, stabilizing the nanosuspension by preparing its IR pellets would be a suitable approach leading to high surface area and increased dissolution rate. Disintegration time and drug dissolution rates of the prepared pellets were investigated. Scanning electron microscopy (SEM) of pellets was carried out to get further insight into the mechanism of dissolution enhancement.

\section{MATERIALS AND METHODS}

\section{Materials}

Tadalafil (Zim Laboratories Ltd., Nagpur, India) was used as a model drug, hydroxypropyl methyl cellulose (HPMC E 15) (Zim Laboratories Ltd., Nagpur, India) and sodium dodecyl sulfate (SDS) (Merck, Mumbai, India) were used as stabilizers to stabilize the nanosuspension. Kcarrageenan (Sigma Life Sciences, Sigma Aldrich, USA) and microcrystalline cellulose (Research Lab Fine Chem Industries, Mumbai) were used as pelletizing agents for preparing immediate release pellets. Sodium starch glycolate (Himedia Laboratories Pvt. Ltd., Mumbai) and crosscarmellose sodium (Research Lab Fine Chem Industries, Mumbai) were used for disintegrating the immediate 
release pellets. Lactose (Himedia Laboratories Pvt. Ltd., Mumbai) was used as a filler to add to the bulk of pellet formulation. Forzest (Ranbaxy, India) was selected as a marketed reference formulation for comparison of dissolution release profiles. All other chemicals and reagents used were of analytical grade.

\section{Preparation of tadalafil nanosuspension}

Nanosuspension containing model drug tadalafil were prepared using high shear homogenization technique [15]. HPMC E 15 and SDS were used in combination as stabilizers. Tadalafil was dispersed in the stabilizer solution and homogenized using high shear homogenizer (Remi-RQT-127A/D, Mumbai, India).

Different batches were prepared by varying the levels of both formulation and process variables such as drug: polymer ratio, drug: surfactant ratio, $\mathrm{d}$ and homogenization time. Composition of different batches of tadalafil nanosuspension are showed in table 1 . The resulting nanosuspension was labelled and stored at room temperature till further evaluation.

Table 1: Composition of formulation batches of Tadalafil Nanosuspension

\begin{tabular}{lllll}
\hline Formulation code & Drug (ratio) & HPMC (ratio) & SDS (ratio) & Homogenizer speed (rpm) \\
\hline NSP1a & 1 & 1 & 1 & 5000 \\
NSP1b & 1 & 1 & 1 & 5000 \\
NSP2a & 1 & 1 & 2 & 5000 \\
NSP2b & 1 & 1 & 2 & 5000 \\
\hline
\end{tabular}

\section{Characterization of tadalafil nanosuspension}

\section{Particle size distribution and polydispersity index (PI)}

Particle size was determined using photon correlation spectroscopy (PCS) [16] using Horiba Nanoparticle Analyser (Nanopartica SZ100). This analysis yields the mean particle diameter (z-average), Polydispersity index and zeta potential at $25^{\circ} \mathrm{C}$.

\section{Zeta potential}

Zeta potential was determined by photon correlation spectroscopy (PCS) using Horiba Nanoparticle Analyser (Nanopartica SZ-100) at $25{ }^{\circ} \mathrm{C}[16]$.

\section{Percent of drug content}

An aliquot $(1 \mathrm{ml})$ of nanosuspension was diluted to $10 \mathrm{ml}$ with methanol and filtered through $0.45 \mu \mathrm{m}$ filter paper. The sample was analyzed using UV-Visible spectrophotometer (Shimadzu-1700, Japan) at $\lambda \max$ of $284 \mathrm{~nm}$ using methanol as blank. Each sample was prepared and analyzed in triplicate.

\section{Saturation solubility studies}

The saturation solubility studies were carried out for both unprocessed pure drug and nanosuspension. Accurately weighed $10 \mathrm{mg}$ of pure drug and nanosuspension equivalent to $10 \mathrm{mg}$ of tadalafil was separately introduced into $25 \mathrm{ml}$ stoppered conical flask containing $10 \mathrm{ml}$ of distilled water. The flask was sealed and placed in a rotary shaker at 37 ${ }^{\circ} \mathrm{C}$ and equilibrated for $6 \mathrm{~h}$. The contents were then filtered, and the suitably diluted samples were analyzed using UV-Visible spectrophotometer (Shimadzu-1700, Japan) at $284 \mathrm{~nm}$, against distilled water as a blank. Each sample was prepared and analyzed in triplicate.

\section{Preparation of immediate release pellets}

Pellets were prepared using the extrusion-spheronization technique [17]. Placebo batch was initially prepared in order to optimize the formula on the basis of evaluation parameters. After optimization of the formula, immediate release pellets of pure drug and immediate release pellets using nanosuspension as a binder were prepared. $\kappa$ carrageenan and microcrystalline cellulose (MCC) were used as pelletizing aids [13]. Sodium starch glycolate and crosscarmellose were used as disintegrants. Amount of the disintegrants to be added was optimized on the basis of preliminary screening. Different concentrations of sodium starch glycolate were used and evaluated for disintegration rate. At lower concentration, disintegration rate was slower and with an increase in the concentration, rapid disintegration was achieved. Above the optimised concentration, time taken for pellets to disintegrate was more. Therefore, an optimized percentage of disintegrant was used in final formulation where faster disintegration of pellets was observed. Lactose was used as a hydrophilic filler. All the ingredients were mixed initially using lab scale blender (AP-01 Plus, Orchid Scientifics, Nashik). For the preparation of IR pellets of pure tadalafil, deionized water was used as a binder whereas other batches of IR pellets of tadalafil were prepared by using nanosuspension as a binder. Each batch contained $50 \mathrm{~g}$ of pellets. The wet mass was extruded using single screw extruder (Anish Pharma Equipments Pvt Ltd.,Nashik) and spheronized for ten minutes at $750 \mathrm{rpm}$ in a spheronizer (Anish Pharma Equipments Pvt Ltd.,Nashik) fitted with cross-hatched rotor plate of $150 \mathrm{~mm}$ diameter and $3 \mathrm{~mm}$ thickness. Resulting pellets were dried at $40{ }^{\circ} \mathrm{C}$ until constant weight. These immediate release pellets were subjected to further evaluation. Composition of different batches of pellets is shown in table 2 and 3 .

Table 2: Composition of placebo batches of IR pellets

\begin{tabular}{|c|c|c|c|c|c|}
\hline Formulation code & к-Carrageenan (\% w/w) & $\begin{array}{l}\text { MCC } \\
(\% \mathrm{w} / \mathrm{w})\end{array}$ & $\begin{array}{l}\text { Sodium starch } \\
\text { Glycolate }(\% \mathrm{w} / \mathrm{w})\end{array}$ & $\begin{array}{l}\text { Crosscarmellose } \\
\text { sodium }(\% \mathrm{w} / \mathrm{w})\end{array}$ & $\begin{array}{l}\text { Lactose } \\
(\% \mathrm{w} / \mathrm{w})\end{array}$ \\
\hline P1 & ---- & 20 & --- & --- & 80 \\
\hline P2 & 20 & 10 & ---- & ---- & 70 \\
\hline P3 & 20 & ---- & ---- & ---- & 80 \\
\hline P4 & 20 & ---- & 7.40 & ---- & 72.6 \\
\hline P5 & 20 & ---- & --- & 7.40 & 72.6 \\
\hline
\end{tabular}

Table 3: Composition of IR pellet batches containing pure drug and nanosuspension

\begin{tabular}{lllll}
\hline Formulation code & $\begin{array}{l}\text { Drug } \\
(\mathbf{\%} \mathbf{w} / \mathbf{w})\end{array}$ & $\begin{array}{l}\boldsymbol{\kappa} \text {-Carrageenan } \\
(\% \mathbf{w} / \mathbf{w})\end{array}$ & $\begin{array}{l}\text { Sodium starch glycolate } \\
(\% \mathbf{w} / \mathbf{w})\end{array}$ & $\begin{array}{l}\text { Lactose } \\
(\% \mathbf{w} / \mathbf{w})\end{array}$ \\
\hline PT1 & 10 & 5 & 7.40 & 47.6 \\
PT2 & 20 & 10 & 7.40 & 62.6 \\
PT3 & 30 & 15 & 7.40 & 77.6 \\
PN* & $30 \mathrm{ml}(25 \mathrm{mg})$ & 20 & 7.40 & q. S. \\
\hline
\end{tabular}

*batch containing nanosuspension as a binder 


\section{Evaluation of IR pellets}

\section{Flow properties}

The flow properties of placebo batches were investigated by measuring the angle of repose, bulk density, tapped density, and Hausner ratio in triplicate by standard official methods.

\section{Drug content}

Accurately weighed $100 \mathrm{mg}$ pellets were finely powdered using mortar-pestle and the powder was dissolved in methanol using sonication. After filtration, the UV absorbance of the suitably diluted filtrate was measured at $\lambda \max 284 \mathrm{~nm}$ to determine the drug content. The determination of drug content was carried out three times for both types of IR pellets. Results are expressed as mean \pm standard deviation.

\section{FTIR study}

Fourier transform infrared (FTIR) studies were carried out to investigate any interaction between pelletizing aid $\kappa$-carrageenan and drug tadalafil. Pure drug, $\kappa$-caragennan and pellets of the formulation containing nanosuspension (PN) were finely powdered, and infrared spectrums of these powders were recorded using FTIR (Shimadzu FTIR-8101) according to the KBr disk method. Chemical integrity of the drug was determined by comparing the infrared (IR) spectra of pellets with that of the pure drug and $\kappa$-carrageenan.

\section{Disintegration time}

The pellet disintegration in water was evaluated by a tablet disintegration tester DT 2 (ROLEX Tablet disintegration rate test apparatus IP). Special transparent tubes of 10-mm diameter and 15$\mathrm{mm}$ length were used. Sieves of $710 \mathrm{~mm}$ mesh size were at the top and the bottom of this tube. After filling $100 \mathrm{mg}$ pellets in each tube, they were inserted in the standard tablet disintegration tester. The disintegration time of six dried samples at $37^{\circ} \mathrm{C}$ was determined at a speed of 30 dips. Disintegration test was carried out three times for both the IR formulations, and Results are expressed as mean \pm standard deviation.

\section{In vitro dissolution studies}

In vitro dissolution studies for both the IR pellets were carried out according to USP 38 basket method for immediate release pellets in $\mathrm{pH} 1.2$ buffer solution,as per the USP general drug release standard for delayed-release dosage form specifications with speed of $75 \mathrm{rpm}$ at $37 \pm 0.5^{\circ} \mathrm{C}$ in $900 \mathrm{ml}$ of dissolution medium. Pellets equivalent to 10 $\mathrm{mg}$ of the drug were weighed and exposed to the dissolution media. At predetermined time intervals, $10 \mathrm{ml}$ of sample was withdrawn and the dissolution medium was kept constant by refilling it with fresh buffer solution to maintain sink conditions. The collected samples were filtered, suitably diluted and analyzed at $284 \mathrm{~nm}$ using UV-Visible spectrophotometer. Dissolution rate profiles of IR pellets were compared with the marketed formulation of tadalafil in order to investigate the potential of IR pellets in improving the dissolution rate.

\section{Scanning electron microscopy}

The surface morphology and cross-section of optimized pellets were characterized using scanning electron microscopy. Photomicrographs of pellets were taken using a scanning electron microscope (Jeol-6380A, Japan Electron Optical Laboratory) for visualization of pellet surface morphology and pellet interior.

\section{RESULTS AND DISCUSSION}

\section{Characterization of tadalafil nanosuspension}

Tadalafil nanosuspensions were prepared using high shear homogenization method. The results obtained from the preliminary evaluation indicates that the formulation NSP2b was clear, transparent and stable in appearance and was selected for further evaluation. Prepared nanosuspension was clear and transparent due to reduced particle size. Effect of homogenization time was studied on the attributes of nanosuspension. Particle size distribution is a very important parameter because reduced particle size helps in the improvement of solubility of pure drug, thereby increasing its dissolution rate. Particle size data and polydispersity index (PI) result for nanosuspension formulation is shown in table 4 .

Table 4: Evaluation of prepared nanosuspension

\begin{tabular}{lllll}
\hline Formulation code & Particle size (nm) & Zeta potential (mV) & Drug content (\%) & PI \\
\hline NSP1a & $445.6 \pm 54.2$ & 22.3 & $92.59 \pm 0.31$ & 0.761 \\
NSP1b & $418.6 \pm 37.4$ & -7.9 & $94.4 \pm 1.06$ & 1.1 \\
NSP2a & $447.7 \pm 29.7$ & -9.4 & $90.45 \pm 0.26$ & 0.568 \\
NSP2b & $247.3 \pm 23.2$ & -11.9 & $98.2 \pm 0.11$ & 0.755 \\
\hline
\end{tabular}

mean $\pm \operatorname{SD}(n=3)$

The mean particle size of different batches of tadalafil nanosuspension was found to be in the range of $245 \mathrm{~nm}$ to $448 \mathrm{~nm}$. Optimized batch (NSP2b) of nanosuspension showed mean particle size of $247.3 \mathrm{~nm}$ at a ratio of 1:1:2 (Drug: Polymer: Surfactant) and with a stirring time of $6 \mathrm{~h}$ at $5000 \mathrm{rpm}$. Fig. 1 shows the particle size distribution of the optimized batch. In general, the decrease in particle size is observed due to increased homogenization/stirring time and also an optimum concentration of suitable stabilizer to prevent agglomeration. A stabilizer is usually incorporated in a nanosuspension formulation to prevent or slow down the crystal growth of nanoparticles via ostwald ripening. Both surfactants and polymers have been widely used as stabilizers for nanosuspensions [18]. Nanosuspensions prepared in this study were stabilized by using both polymer HPMC and surfactant SDS in combination. In comparison with polymers, surfactants can provide more effective wetting of the drug particles. This would result in better dispersion of the drug particles and a greater extent of particle size reduction. Amount of surfactant played a significant role in size reduction process as evident from the results. SDS is an ionic surfactant and in this study was used as a stabilizer for charged stabilization of the homogenized and suspended nanoparticles. As the amount of surfactant increased, the particle size also reduced drastically from $418 \mathrm{~nm}$ to $247 \mathrm{~nm}$. Also, an increased amount of SDS might have resulted into stabilization of more number of particles as evident from the polydispersity index which is 0.755 in case of NSP2b and 1.1 in case of NSP1b. This can be attributed to the good wetting property and dispersibility exhibited by the surfactant used in the above ratio. SDS at the above concentration yielded a stable nanosuspension with zeta potential in the range and also showed reduced particle size to a greater extent. Therefore, the above concentration of the stabilizer was optimized. Impact of further higher ratios of surfactant on the particle size reduction and stability was not explored and can be further investigated. It seems reasonable to postulate that use of optimum concentration of suitable stabilizer and stirring rate results in fine nanosuspension with smaller and more uniform particle size [15].

Determination of zeta potential is essential as it indicates the physical stability of the colloidal dispersion. Particle charge governs the physical stability of the nanosuspension and therefore is one of the most important evaluation parameters. The nanosized particles possess high surface energy and are prone to agglomeration or aggregation of drug crystals. Stabilizers prevent agglomeration or aggregation to yield a physically stable formulation by providing steric or ionic barriers [19]. The zeta potential of a nanosuspension depends upon the stabilizer and the 
drug itself. A minimum zeta potential of $\pm 30 \mathrm{mV}$ is required for an electrostatically stabilized nanosuspension whereas a minimum zeta potential of $\pm 20 \mathrm{mV}$ is desirable in the case of a combined electrostatic and steric stabilization $[16,19]$. In order to obtain a nanosuspension exhibiting good stability, prepared nanosuspension was stabilized using a combination of polymer and surfactant. Combination of these two types of stabilizers imparts steric as well as electrostatic stabilization to the system [20]. All the batches of nanosuspension showed zeta potential values in the range- $7.9 \mathrm{mV}$ to $22.3 \mathrm{mV}$, indicating physically stable systems. Use of HPMC and SDS successfully prevents aggregation of the nano-sized particles.

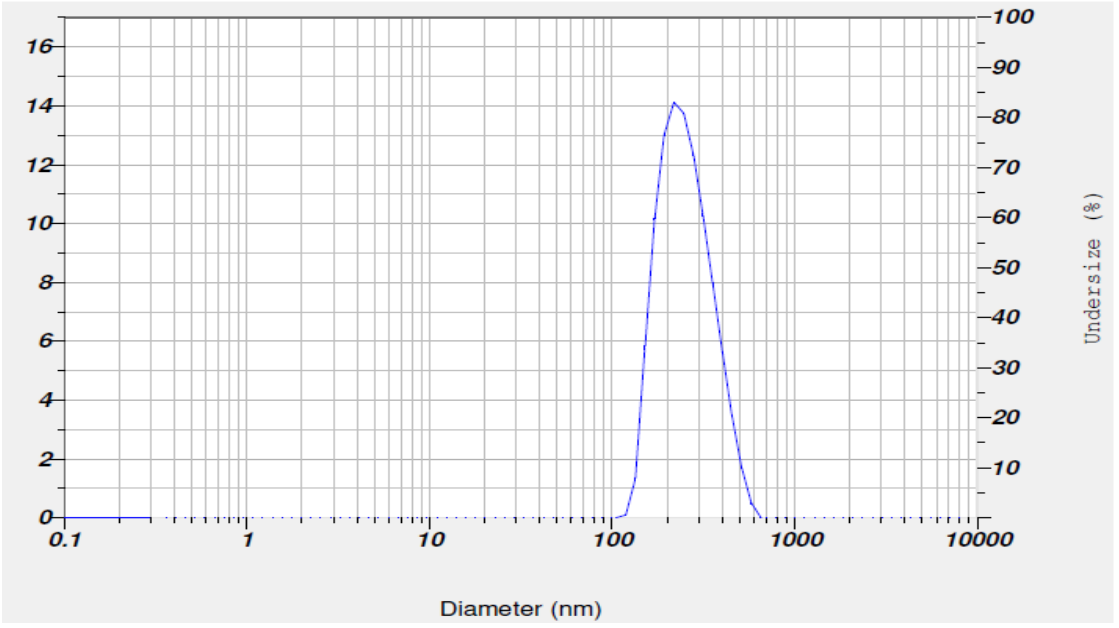

Fig. 1: Particle size distribution of NSP2b

The drug content was determined by UV-visible spectrophotometer method. Formulation NSP2b was found to contain the highest drug content amongst the other formulations.

The saturation solubility data of tadalafil nanosuspension NSP2b and the pure drug was found to be 7.4 and $0.133 \mathrm{mg} / \mathrm{ml}$ as shown in table 5 . The result indicates that nanosuspension showed maximum solubility compared to pure drug. In these studies, the aqueous solubility of tadalafil nanosuspension was found to be greater than pure tadalafil. The reduction of particle size of the homogenized drug to the nano range increased the surface area and use of hydrophilic polymer as a stabilizer was responsible to enhance the saturation solubility.

Table 5: Saturation solubility data of tadalafil nanosuspension and pure drug

\begin{tabular}{lll}
\hline Formulation code & Formulation type & Saturation solubility $(\mathrm{mg} / \mathrm{ml})$ \\
\hline NSP2b & Nanosuspension & $\mathbf{7 . 4 \pm 1 . 4 6}$ \\
-- & Pure drug & $0.133 \pm 0.39$ \\
\hline
\end{tabular}

mean $\pm \operatorname{SD}(n=3)$

\section{Evaluation of immediate release pellets}

Results of the investigation of flow properties indicates good and excellent pellet properties for all the placebo batches. Addition of $\kappa$ - carrageenan as a pelletizing agent results in the improvement of flow. Table 6 shows the data for flow properties and drug content of the prepared immediate release pellets of pure drug and nanosuspension.

Table 6: Flow properties of IR pellets containing pure drug and nanosuspension

\begin{tabular}{|c|c|c|c|c|c|c|c|}
\hline $\begin{array}{l}\text { Formulation } \\
\text { code }\end{array}$ & Angle of repose $\left({ }^{0}\right)$ & $\begin{array}{l}\text { Bulk density } \\
(\mathrm{g} / \mathrm{ml})\end{array}$ & $\begin{array}{l}\text { Tapped density } \\
(\mathrm{g} / \mathrm{ml})\end{array}$ & Hausner ratio & $\begin{array}{l}\text { Carr's index } \\
(\%)\end{array}$ & $\begin{array}{l}\text { Friability } \\
(\%) \\
\end{array}$ & $\begin{array}{l}\% \\
\text { drug content }\end{array}$ \\
\hline PT1 & $26.39 \pm 0.05$ & $0.67 \pm 0.004$ & $0.74 \pm 0.010$ & $1.10 \pm 0.02$ & $9.30 \pm 0.12$ & $0.04 \pm 0.2$ & $92.42 \pm 0.24$ \\
\hline PT2 & $27.79 \pm 0.065$ & $0.68 \pm 0.032$ & $0.75 \pm 0.028$ & $1.05 \pm 0.16$ & $5.47 \pm 0.32$ & $0.018 \pm 0.1$ & $94.93 \pm 0.07$ \\
\hline PT3 & $25.59 \pm 0.09$ & $0.77 \pm 0.021$ & $0.85 \pm 0.043$ & $1.10 \pm 0.23$ & $8.30 \pm 0.09$ & $0.03 \pm 0.6$ & $95.33 \pm 0.15$ \\
\hline $\mathrm{PN}$ & $29.33 \pm 0.32$ & $0.69 \pm 0.008$ & $0.72 \pm 0.004$ & $1.04 \pm 0.06$ & $4.10 \pm 0.05$ & $0.02 \% \pm 0.4$ & $99.61 \pm 0.41$ \\
\hline
\end{tabular}

mean $\pm \operatorname{SD}(n=3)$

Disintegration study revealed that the use of different pelletizing agents and disintegrants affects the process of disintegration. MCC used alone as a pelletizing agent showed a slow disintegration process with a higher disintegration time of 16 min (formulation $\mathrm{P} 1)$, also as the concentration of MCC reduced, disintegration time also decreased. Addition of $\kappa$-carrageenan reduced the time from 16 min to around $11 \mathrm{~min}$ (formulation P2). While in case of other pelletizing agent $\kappa$-carrageenan alone, disintegration time was found to be reduced to 4 min 22 seconds (formulation P3). Addition of disintegrants further lowered the disintegration time. With sodium starch glycolate, disintegration time was lowest (formulation P4), indicating very rapid disintegration of pellets. The order of disintegration for different batches was $\mathrm{P} 1>\mathrm{P} 2>\mathrm{P} 3>\mathrm{P} 5>\mathrm{P} 4$. Use of $\kappa$ carrageenan as a pelletizing agent offers more wettability to the system resulting in a faster rate of diffusion of the dissolution medium into the pellet interior [21]. Disintegration studies become 
important for immediate release pellets, where faster drug release is required in gastrointestinal fluids. Faster disintegration will result into more number of smaller particles in the dissolution medium. This will result in a great increase in surface area and increased water solubility. Rapid disintegration of pellet formulation P4 must be because of swelling of $\kappa$-carrageenan and sodium starch glycolate in the presence of disintegration medium. Placebo batch P4 was selected for preparing IR pellets containing pure drug and using nanosuspension as a binder. Disintegration pattern of IR pellets of the pure drug showed a slight increase in disintegration time with an increase in the concentration of the drug. Nanosuspension containing pellets showed the fastest disintegration amongst all other pellet batches. Presence of drug in nanosize in oral multiparticulate dosage form added to the already enhanced surface area of IR pellets by virtue of smaller particle size and presence of HPMC and SDS might have contributed to faster disintegration. No significant difference in disintegration time was observed within the batches of IR pellets. Table 7 shows disintegration time for various batches of IR pellets.

Table 7: Disintegration time for various formulation batches of pellets

\begin{tabular}{|c|c|c|c|}
\hline Placebo IR pellet batches & Disintegration time & Tadalafil IR pellet batches & Disintegration time \\
\hline P1 & $16 \min 05 \mathrm{sec} \pm 0.04$ & PT1 & $2 \min 28 \sec \pm 0.12$ \\
\hline P2 & $10 \min 54 \sec \pm 0.16$ & PT2 & $2 \min 52 \sec \pm 0.32$ \\
\hline P3 & $4 \min 22 \sec \pm 0.06$ & PT3 & $3 \min 12 \sec \pm 0.05$ \\
\hline P4 & $1 \mathrm{~min} 49 \mathrm{sec} \pm 0.02$ & PN & $2 \min 13 \mathrm{sec} \pm 0.04$ \\
\hline P5 & $2 \min 34 \mathrm{sec} \pm 0.09$ & & \\
\hline
\end{tabular}

mean \pm SD $(n=3)$

\section{FTIR study}

The FTIR spectra of tadalafil pellets prepared using $\kappa$-carrageenan when compared with IR spectra of tadalafil and $\kappa$-carrageenan showed no interaction of tadalafil with $\kappa$-carrageenan, indicating the compatibility of the drug with the excipient. Hence, $\kappa$-carrageenan can be retained as an excipient. Principle peaks of tadalafil and $\kappa$ carrageenan can be seen in the IR spectra of pellets prepared using $\kappa$-carrageenan. Fig. 2 shows the IR spectra of drug, excipient and formulation. From the spectra, it can be concluded that tadalafil remains unaffected during the process of extrusion and spheronization in the presence of $\kappa$-carrageenan.

\section{Spectrum}

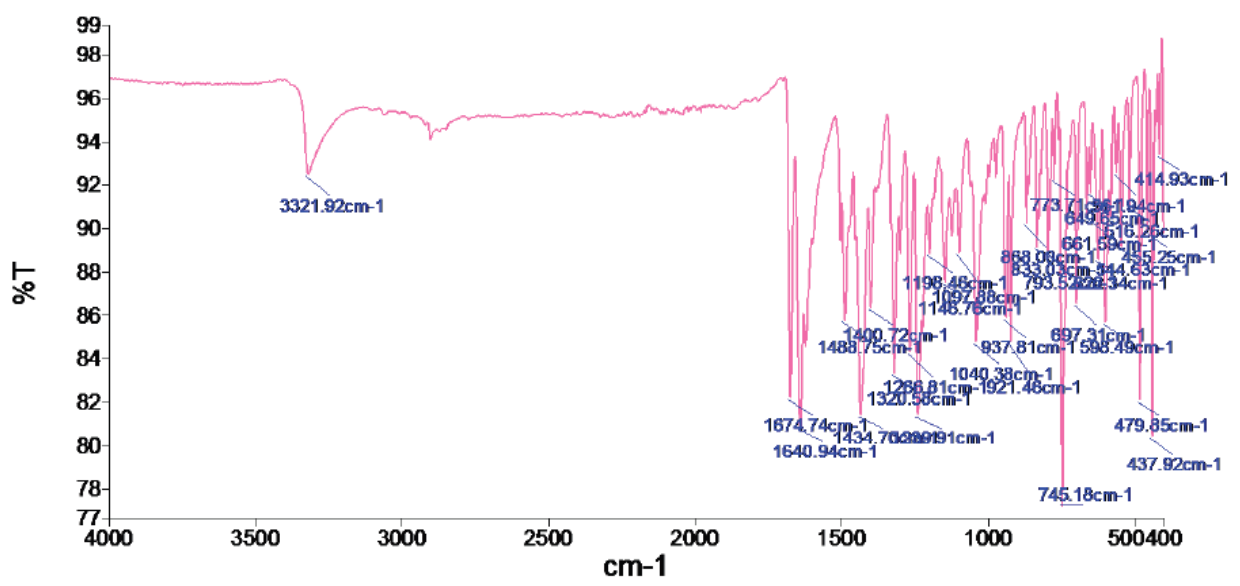

Fig. 2: (a) FTIR spectra of tadalafil

\section{Spectrum}

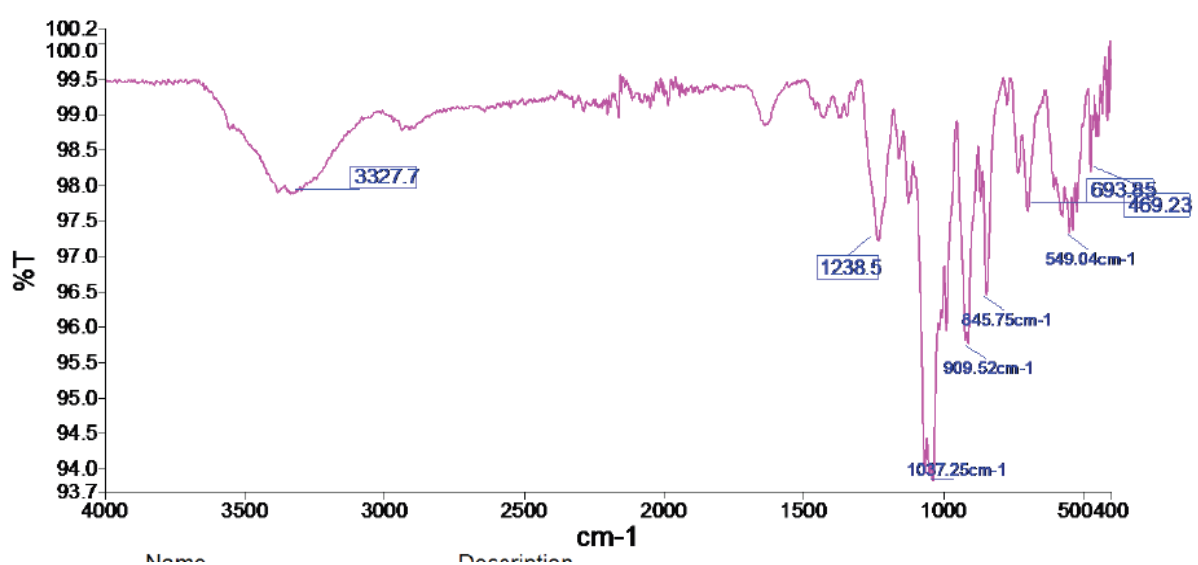

Fig. 2: (b) FTIR spectra of $\kappa$-carrageenan 


\section{Spectrum}

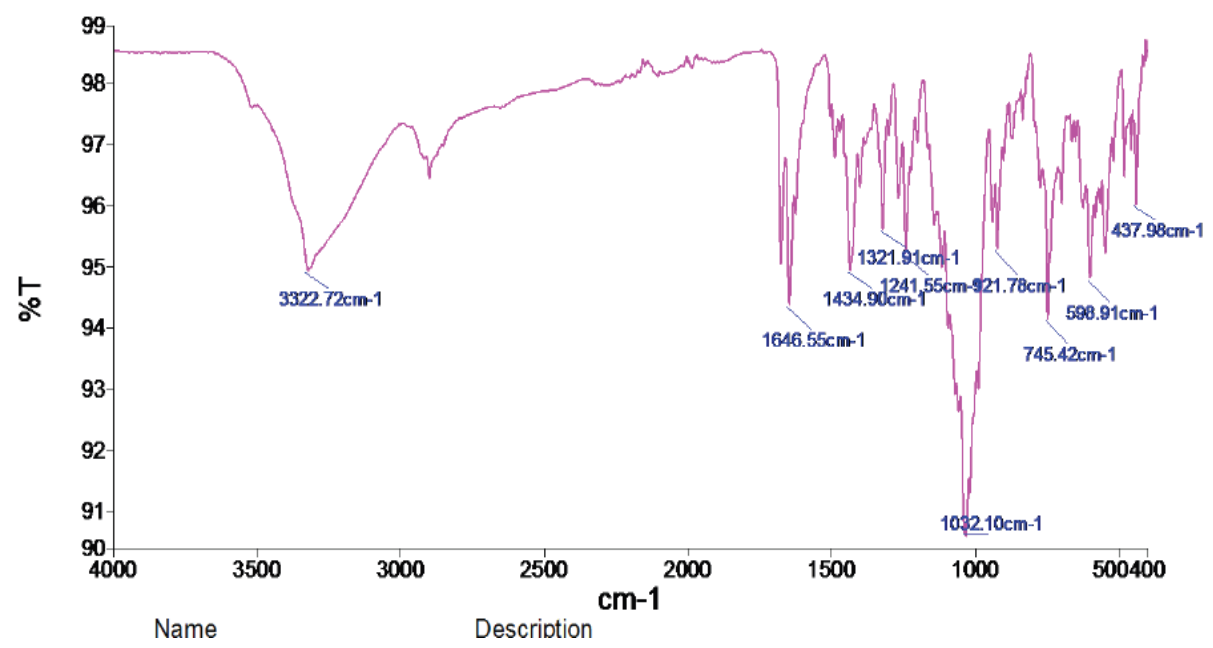

Fig. 2: (c) FTIR spectra of pellets of tadalafil and $\kappa$-carrageenan

\section{In vitro dissolution studies}

The dissolution profile of IR pellets of pure drug and pellets prepared using nanosuspension as a binder were compared with pure drug and marketed tablets of tadalafil. It was observed that pellets prepared using nanosuspension (PN) released more than $85 \%$ drug within 45 min whereas IR pellets of the pure drug (PT1) released about $70 \%$ drug within $45 \mathrm{~min}$ as compared to the marketed tablet which released only $66 \%$ drug within 45 min. Fig. 3 shows the data of dissolution studies carried out for different batches. Dissolution of the drug depends on its release from the dosage form, which in turn depends on the disintegration of oral formulation. Drugs with poor solubility faces the issue of poor dissolution and absorption after release from the dosage form. This accounts for poor bioavailability of the drug. Faster disintegration and increase in surface area at the site of dissolution can lead to better and enhanced contact of drug molecules with the physiological fluid and can give a faster rate of dissolution. This combined approach can be achieved by preparing immediate release multiparticulate dosage form of the drug. IR pellets of the drug were prepared with the objective of enhanced dissolution. Significant increase was found in drug release from IR pellets as compared to pure drug and marketed formulation.

Solidification approach of nanosuspension by using it as a binder in IR pellets resulted in a marked increase in the drug release pattern, which can be due to the synergistic effect produced by the reduced particle size at nano level and delivery of the poorly soluble drug in the form of IR pellets. Therefore, it can be concluded that both the approaches i.e. use of nanosuspension as a binder and use of $\kappa$ carrageenan for IR pellets improved the dissolution rate of tadalafil. Results are expressed as the mean of three determinations.

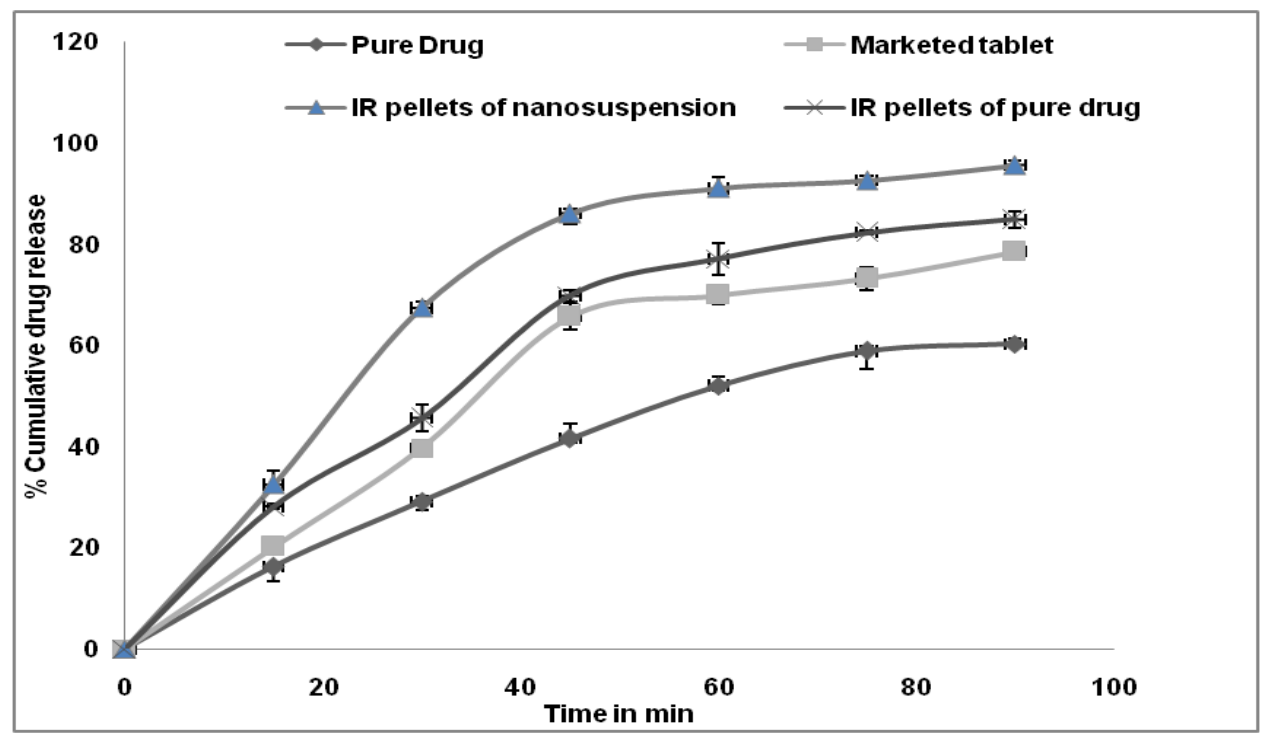

Fig. 3: Release profiles of different batches of immediate release pellets

\section{Scanning electron microscopy}

Scanning electron micrographs of IR pellets prepared using nanosuspension as a binder are shown in fig. 4. The selected formulation (PN) was chosen on the basis of results obtained from the disintegration studies and dissolution studies. Fig. 4a shows the surface morphology of pellets with a slightly rough surface. Fig. $4 \mathrm{~b}$ and $4 \mathrm{c}$ shows a cross-section of pellets, where the interior of the pellet seems to be porous in nature. The pellets prepared using $\kappa$ carrageenan as pelletizing agents were perfectly spherical and smooth with fine pores in it. The cross-section of the pellet shows porous interior, responsible for the rapid disintegration of the pellet. 


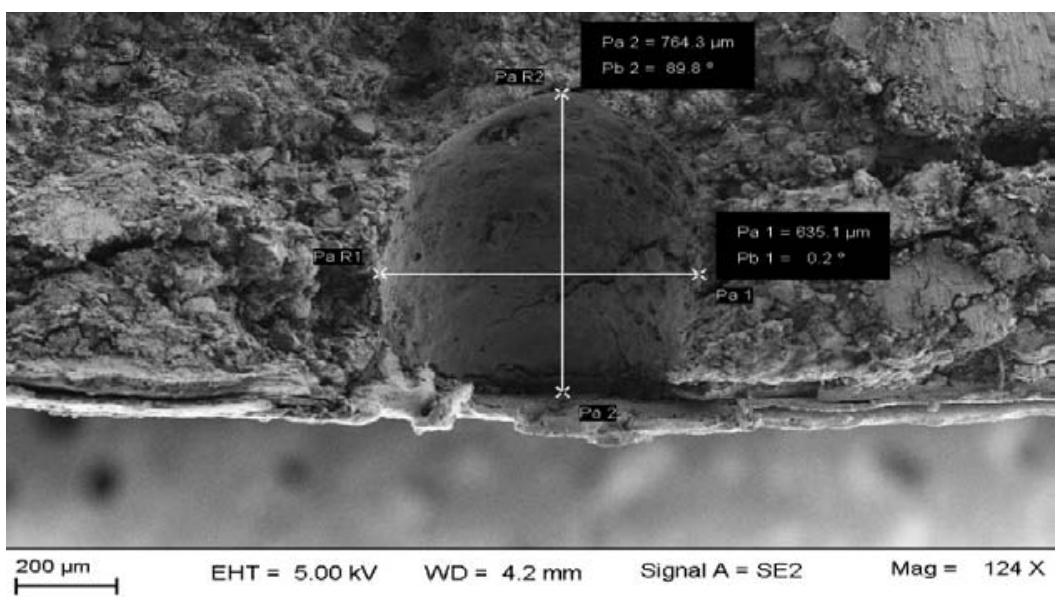

Fig. 4: (a) Scanning electron micrograph showing surface morphology of pellets

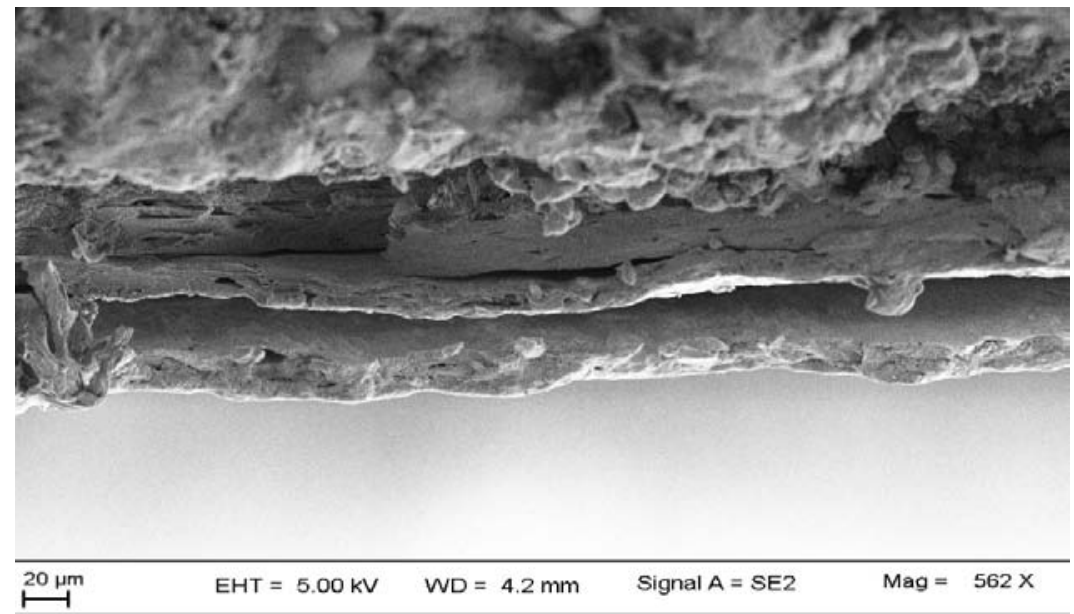

Fig. 4: (b) Scanning electron micrograph showing the cross section of pellets

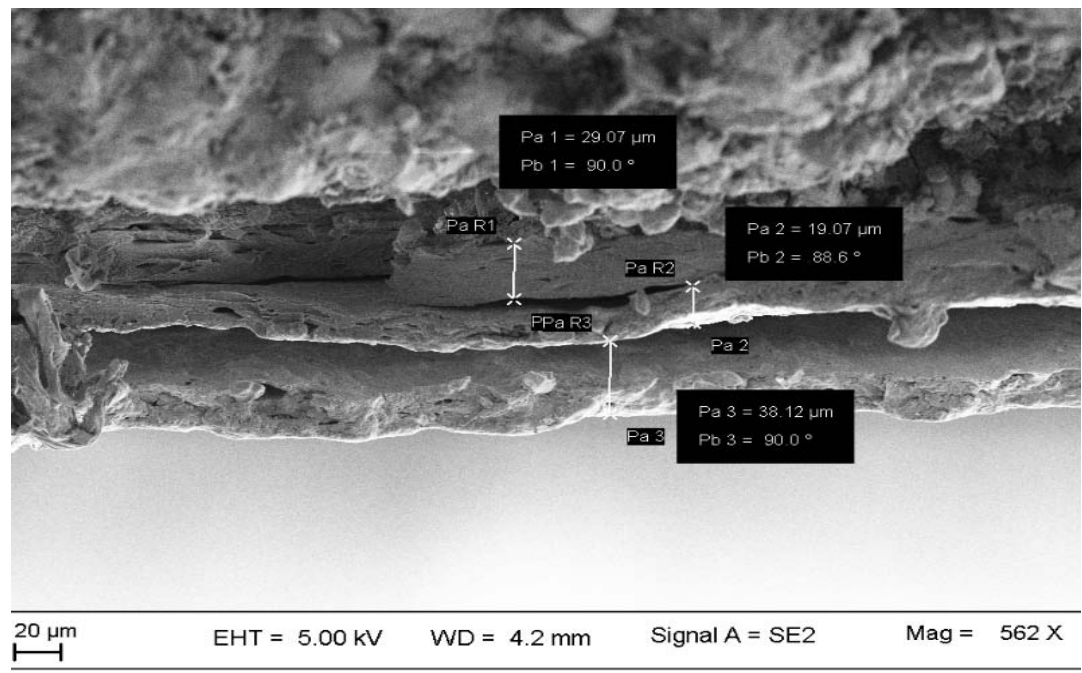

Fig. 4: (c) Scanning electron micrograph showing cross section of pellets

\section{Stability studies of IR pellets containing nanosuspension}

Stability data revealed no major changes in the desired characters of pellets. Drug content, disintegration time and dissolution study showed satisfactory results. During the storage period, pellets retained the original good flowing properties. Table 8 shows the results of stability data of IR pellets containing nanosuspension. 
Table 8: Stability studies data of IR pellets containing nanosuspension

\begin{tabular}{llll}
\hline Evaluation parameters & $\mathbf{0 ~ m o}$ & $\mathbf{1 ~ m o}$ & 2 mo \\
\hline Drug content & $99.61 \pm 0.48$ & $99.58 \pm 0.35$ & $99.53 \pm 0.008$ \\
Disintegration time & 2 min $13 \mathrm{sec} \pm 0.08$ & $2 \mathrm{~min} 15 \mathrm{sec} \pm 0.20$ & $2 \mathrm{~min} 14 \mathrm{sec} \pm 0.48$ \\
Angle of repose & $29.33 \pm 0.32$ & $29.7 \pm 0.08$ & $29.06 \pm 0.04$ \\
\% Drug release & $95.66 \pm 0.008$ & $95.01 \pm 0.004$ & $94.67 \pm 0.008$ \\
\hline
\end{tabular}

mean $\pm \operatorname{SD}(n=3)$

\section{CONCLUSION}

From the above investigation, it can be concluded that the formulation of Immediate-release pellets using nanosuspension as a binder can be a novel and feasible option for stabilization as well as solidification of nanosuspensions. Use of high shear homogenizer as a non-specific technique for size reduction can produce nanosuspension with significant improvement in dissolution rate. The study has revealed that it is possible to enhance the solubility of tadalafil by preparing its nanosuspension using high shear homogenization technique. Further, the solubility of drug can be improved by formulating IR pellets using $\kappa$-carrageenan for improvement of dissolution rate. Solidification of nanosuspension in the form of pellets can be further explored for increasing the solubility of other poorly soluble drugs and stabilization of nanosuspension.

\section{ACKNOWLEDGMENT}

Authors would like to acknowledge Dr. A. M. Ittadwar, Principal, Gurunanak College of Pharmacy, Nagpur for providing infrastructural facilities to carry out the research work. We are also thankful to Dr. Vipin Dhote, Principal VNS Institute of Pharmacy, Bhopal, India for providing facilities for carrying out particle size analysis using Horiba Nanosizer and Zim Laboratories Ltd. Kalmeshwar, Nagpur for providing gift sample of API tadalafil and polymer HPMC E15.

\section{AUTHORS CONTRIBUTIONS}

All the authors have contributed equally

\section{CONFLICT OF INTERESTS}

The authors report no conflicts of interest. The authors alone are responsible for the content and writing of this article

\section{REFERENCES}

1. Javiana L, Teobaldo A, Jacqueline S, Hernandez L, Von Plessing C. Preliminary pharmacokinetic study of different preparations of acyclovir with b-cyclodextrin. J Pharm Sci 2002;91:2593-8.

2. Norbert R, Muller BW. Dissolution rate enhancement by in situ micronization of poorly water-soluble drugs. Pharm Res 2002;19:1894-900.

3. Hajare AA, Jadhav PR. Improvement of solubility and dissolution rate of indomethacin by solid dispersion in polyvinyl pyrrolidone K30 and poloxomer 188. Asian J Pharm Technol 2012;2:116-22.

4. Stella VJ, He Q. Cyclodextrins. Toxicol Pathol 2008;36:30-42.

5. Asare Addo K, Supuk E, Al Hamidi H, Owusu Ware S, Nokhodchi A, Conway BR. Triboelectrification and dissolution property enhancements of solid dispersions. Int J Pharm 2015;485:306-16.

6. Laxmi P, Ashwinikumar G. Nanosuspension technology: a review. Int J Pharm Pharm Sci 2010;(2, Suppl 4):35-40.

7. Baek I, Kim J, Eun Sol Ha, Gwang Ho Choo. Dissolution and oral absorption of pranlukast nanosuspensions stabilized by hydroxypropylmethylcellulose. Int J Biol Macromol 2014;67:53-7.

8. Van Eerdenbrugh B, Froyen L, Van Humbeeck J, Martens JA, Augustijns P, Van den Mooter G. Drying of crystalline drug nanosuspensions-The importance of surface hydrophobicity on dissolution behavior upon redispersion. Eur J Pharm Sci 2008;35:127-35.

9. He W, Lu Y, Qi J, Chen L, Yin L, Wu W. Formulating food proteinstabilized indomethacin nanosuspensions into pellets by fluidbed coating technology: physical characterization, redispersibility, and dissolution. Int J Nanomed 2013;8:3119-28.

10. Singare DS, Marella S, Gowthamrajan K, Kulkarni GT, Vooturi R, Rao PS. Optimization of formulation and process variable of nanosuspension: an industrial perspective. Int J Pharm 2010;402:213-20

11. Ghebre Sellassie. Pharmaceutical pelletization technology. New York: Marcel Dekker; 1989.

12. Nassab PR, Rajko R, Szabo Revesz P. Physicochemical characterization of meloxicam-mannitol binary systems. J Pharm Biomed Anal 2006;41:1191-7.

13. Thommes $M$, Kleinebudde $P$. Use of $\kappa$-carrageenan as alternative pelletization aid to microcrystalline cellulose in extrusion/spheronization, I: influence of type and fraction of filler. Eur J Pharm Biopharm 2006;63:59-67.

14. Brock GB, McMahon CG, Chen KK, Costigan T, Shen W, Watkins V. Efficacy and safety of tadalafil for the treatment of erectile dysfunction: results of integrated analyses. J Urol 2002;168:1332-6.

15. Kilor V, Sapkal N, Daud A, Humne S, Gupta T. Development of stable nanosuspension loaded oral films of glimepiride with improved bioavailability. Int J Appl Pharm 2017;9 Suppl 2:28-33.

16. Patravale VB, Date AA, Kulkarni RN. Nanosuspensions: a promising drug delivery strategy. J Pharm Pharmacol 2004;56 Suppl 7:827-40.

17. Kilor VA, Sapkal NP, Awari JG, Shewale BP. Development and characterization of enteric-coated immediate-release pellets of aceclofenac by extrusion/spheronization technique using $\kappa$ carrageenan as a pelletizing agent. AAPS PharmSciTech 2010;11 Suppl 1:336-43.

18. Lee J, Lee SJ, Choi JY, Yoo JY, Ahn CH. Amphiphilic amino acid copolymers as stabilizers for the preparation of nanocrystal dispersion. Eur J Pharm Sci 2005;24:441-9.

19. Bilgili E, Li M, Afolabi A. Is the combination of cellulosic polymers and anionic surfactants a good strategy for ensuring physical stability of BCS Class II drug nanosuspensions? Pharm Dev Technol 2016;21 Suppl 4:499-510.

20. Muller RH, Jacobs C, Kayser O. Nanosuspensions as particulate drug formulations in therapy: rationale for the development and what we can expect for the future. Adv Drug Delivery Rev 2001;47:3-19.

21. Thommes $\mathrm{M}$, Kleinebudde $\mathrm{P}$. Use of $\kappa$-carrageenan as alternative pelletization aid to microcrystalline cellulose in extrusion/spheronization, II: influence of drug and filler type. Eur J Pharm Biopharm 2006;63:68-75. 